- Mandibular advancement splints can be successfully used in the treatment of patients with sleep-related breathing disorders.

- Patients presenting with obstructive sleep apnoea can have a wide range of disease severity, which may not be indicative of treatment outcome.

- Follow-up sleep studies are required.

\title{
The effect of a mandibular advancement splint in subjects with sleep-related breathing disorders
}

\author{
A. Johal, ${ }^{1}$ D. Arya, ${ }^{2}$ L. J. Winchester, ${ }^{3}$ P. J. H. Venn ${ }^{4}$ and H. Brooks ${ }^{5}$
}

\begin{abstract}
Objective This study assessed the effectiveness of a mandibular advancement splint (MAS) in subjects with sleep-related breathing disorders using both objective and subjective outcome measures. Design The study was carried out as a retrospective analysis. Setting The study was conducted within the Sleep Studies Unit at the Queen Victoria Hospital, East Grinstead, between May 1997 and March 2000.
\end{abstract}

Subjects and methods Twenty subjects with obstructive sleep apnoea (OSA) and six with non-apnoeic snoring, diagnosed by overnight polysomnography, were fitted with a monobloc appliance between May 1997 and March 2000.

Main outcome measures The subjects were analysed for changes in the respiratory disturbance index (RDI) and Epworth Sleepiness Scale (ESS) scores. In addition each subject completed an outcome questionnaire following fitting of the appliance.

Results Variability in response measured by the change in the respiratory disturbance index was found with no correlation to the baseline recording. Although median RDI values improved in both groups, significantly so in the obstructive sleep apnoea group $(p<0.05)$, seven subjects exhibited an increased RDI score following mandibular advancement splint therapy. The median Epworth Sleepiness Scale scores decreased in both the OSA group and the non-apnoeic snorers although not significantly. Twenty-one of the 26 subjects completed the outcome questionnaire revealing an $81 \%$ reduction in snoring. Side-effects were generally transient and minor. Eighty-six per cent of the subjects' partners reported better quality of sleep as a result of MAS therapy. Conclusions The monobloc appliance significantly improved the Respiratory Disturbance Index in the obstructive sleep apnoea group. Some subjects had increased RDI scores following splint therapy. This supports the need for an objective assessment in the follow-up of patients treated with mandibular advancement splints.

\footnotetext{
1*Senior Clinical Lecturer, Department of Orthodontics, Institute of Dentistry, St. Bartholomew's and The Royal London School of Medicine and Dentistry, New Road, Whitechapel, London E1 1BB; ${ }^{2}$ Specialist Practitioner, ${ }^{3}$ Consultant Orthodontist, Department of Orthodontics, Queen Victoria Hospital, East Grinstead; ${ }^{4}$ Consultant Anaesthetist, Department of Anaesthetics, Queen Victoria Hospital, East Grinstead; ${ }^{5}$ Sleep Technician, Queen Victoria Hospital, East Grinstead

${ }^{*}$ Correspondence to: Dr A. Johal

Email:a.s.johal@qmul.ac.uk
}

\section{Refereed paper}

Accepted 13 April 05

doi: $10.1038 /$ sj.bdj.4812903

$\odot$ British Dental Journal 2005; 199: 591-596

\section{INTRODUCTION}

Sleep-related abnormalities of respiration have been reported since the mid-nineteenth century. ${ }^{1}$ Simple snorers are patients with loud snoring in the absence of any other abnormal breathing episodes such as apnoeas or hypopnoeas. ${ }^{2}$ Apnoea is defined as the complete cessation of breathing lasting at least 10 seconds. ${ }^{3}$ Hypopnoea is a milder form of defined breathing disturbance with a 50\% reduction in ribcage and abdominal excursion for at least 10 seconds, with an accompanying dip of $4 \%$ in oxygen saturation. ${ }^{4}$ Thus, an overnight sleep study, such as polysomnography, is regarded as the gold standard for the diagnosis of a sleep-related breathing disorder, permitting the distinction between simple snoring and obstructive sleep apnoea (OSA). The severity of obstructive sleep apnoea can be expressed by the apnoea/hypopnoea index (AHI) or the respiratory disturbance index (RDI) per hour of sleep.

Recently, the American Academy of Sleep Medicine Task Force ${ }^{5}$ defined mild obstructive sleep apnoea as an apnoea/hypopnoea index of five to 15 events per hour, moderate OSA as an AHI of 1630 events per hour and severe OSA as an AHI greater than 30 events per hour of sleep.

Ohayon et al. ${ }^{6}$ reported the prevalences of snoring in the UK as being $40 \%$ in their population sample. Gibson et al. ${ }^{7}$ report obstructive sleep apnoea to affect one to two per cent of males in the UK and approximately half this number of females.

The clinical features of obstructive sleep apnoea can be conveniently divided into those occurring during sleep and include snoring, choking, abnormal motor activity and nocturia; and those occurring during wakefulness of which excessive daytime sleepiness is the most common. Complications of obstructive sleep apnoea include hypertension, cerebrovascular and cardiovascular disease although divergence exists in the evidence. ${ }^{8}$

Overnight polysomnography combines electrophysiological indices of sleep stage, electromechanical parameters contrasting respiratory effort with actual ventilation, and measurements reflecting the consequences of any abnormal respiratory events. Mini-sleep studies have been introduced as a method of overcoming these limitations and still offering the relevant information. ${ }^{9}$

Various tests exist to measure sleepiness although confusion exists about what sleepiness is and what these tests actually measure. ${ }^{10}$ The Epworth Sleepiness Scale (ESS) is a validated simple questionnaire that asks the subject to rate on a scale of 0 to 3 the chances that, as part of his or her 'usual way of life in recent times' s/he would doze in each of eight different situations. ${ }^{11}$ The latter 


\begin{tabular}{|c|c|c|c|c|}
\hline \multirow[b]{2}{*}{ Variable } & \multicolumn{2}{|c|}{$\begin{array}{l}\text { OSA }^{+} \text {subjects } \\
(n=20) \\
M=15 ; F=5\end{array}$} & \multicolumn{2}{|c|}{$\begin{array}{l}\text { Non-apnoeic snorers } \\
\qquad \begin{array}{l}(n=6) \\
M=6 ; F=0\end{array}\end{array}$} \\
\hline & Median & Range & Median & Range \\
\hline Age & 53.1 & $37.3-66.8$ & 46.8 & $41.1-57.8$ \\
\hline BMI & 27.0 & $20-33$ & 28.0 & $25-34$ \\
\hline $\begin{array}{l}\mathrm{RDI}^{*} \\
\text { (pre- } \\
\mathrm{MAS}^{* *} \text { ) }\end{array}$ & 8.0 & $6-65$ & 2.0 & $1-3$ \\
\hline $\begin{array}{l}\text { ESS }^{* * *} \\
\text { (pre- } \\
\text { MAS**) }^{* *}\end{array}$ & 10.0 & $1-18$ & 12.5 & $8-20$ \\
\hline
\end{tabular}

${ }^{+}$Obstructive Sleep Apnoea *Respiratory Disturbance Index ** Mandibular advancement splint ***Eworth Sleepiness scale score

\begin{tabular}{|c|c|c|c|c|}
\hline \multirow{2}{*}{$\begin{array}{l}\text { Timing } \\
\text { of sleep } \\
\text {-study }\end{array}$} & \multicolumn{3}{|c|}{ Sleep-related breathing disorder } & \multirow[b]{2}{*}{$\begin{array}{c}\text { Severe OSA } \\
\mathrm{RDI}^{+}>30\end{array}$} \\
\hline & $\begin{array}{c}\text { Non-apnoeic } \\
\text { snorer } \\
\mathrm{RDI}^{+}<5\end{array}$ & $\begin{array}{l}\text { Mild OSA } \\
\text { RDI }^{+} 5-15\end{array}$ & $\begin{array}{l}\text { Mod. OSA } \\
\text { RDI }^{+} 16-30\end{array}$ & \\
\hline Baseline & 6 & 9 & 5 & 6 \\
\hline $\begin{array}{l}\text { Following } \\
\text { MAS therapy }\end{array}$ & 13 & 6 & 4 & 3 \\
\hline
\end{tabular}

\begin{tabular}{|c|c|c|c|c|c|c|}
\hline \multirow[t]{2}{*}{ Variable } & \multicolumn{2}{|c|}{ OSA subjects } & \multirow[t]{2}{*}{$P$} & \multicolumn{2}{|c|}{ Non-apnoeic snorers } & \multirow[t]{2}{*}{$P$} \\
\hline & Median & Range & & Median & Range & \\
\hline $\begin{array}{l}\text { RDI } \\
\text { (pre-MAS) }\end{array}$ & 18.0 & $6-65$ & * & 2.0 & $1-3$ & Ns \\
\hline $\begin{array}{l}\text { RDI } \\
\text { (post-MAS } \neq)\end{array}$ & 7.0 & $0-72$ & & 1.0 & $0-13$ & \\
\hline $\begin{array}{l}\text { ESS } \\
\text { (pre-MAS) }\end{array}$ & 10.0 & $1-18$ & Ns & 12.5 & $8-20$ & Ns \\
\hline $\begin{array}{l}\text { ESS } \\
\text { (post-MAS) }\end{array}$ & 9.0 & $0-17$ & & 8.5 & $5-14$ & \\
\hline
\end{tabular}

were chosen on a priori grounds to vary in their soporific nature from highly soporific ("lying down to rest in the afternoon when circumstances permit') to much less soporific ('sitting and talking to someone'). The ESS score is the sum of eight item scores and can range from 0 to 24. The clinical 'normal' range of scores is 2 to 10 with a mode score of six. ${ }^{10}$ The relationship between the severity of obstructive sleep apnoea and daytime sleepiness, as assessed by the ESS questionnaire, remains controversial. Johns ${ }^{10}$ demonstrated that the scores increase linearly with the severity of obstructive sleep apnoea and can distinguish primary snorers from patients with obstructive sleep apnoea, even of mild degree. These findings are in contrast to those by Osman et al. ${ }^{12}$ who failed to show a correlation between apnoea/hypopnoea index and Epworth sleepiness scale scores. The authors suggested this variance to be due to simple snorers suffering from excessive daytime sleepiness as a result of an unclear mechanism and that the ESS is of use in assessing the disability as a result of snoring but of no value in screening obstructive sleep apnoea patients among snorers.

The treatment objectives for patients with sleep-related breathing disorders should be to alleviate snoring in simple snorers and to resolve the clinical signs and symptoms in patients with obstructive sleep apnoea with normalisation of the respiratory disturbance index and oxyhaemoglobin saturation. ${ }^{13}$ For obstructive sleep apnoea, no currently available treatment provides the ideal combination of high rate success and patient compliance without complications. As a consequence a number of diverse treatment approaches have been proposed which can be broadly divided into surgical and non-surgical therapies. ${ }^{14}$ Surgical therapies include tracheostomy, nasal surgery, maxillofacial surgery, base of tongue resection, hyoid bone repositioning and pharyngeal surgery. Nonsurgical therapies include eliminating aggravating factors, electrical stimulation of the upper airway, pharmacological therapy, nasally applied continuous positive airway pressure (CPAP) and intra-oral appliances. The latter comprise tongue retaining devices and the more widely used mandibular advancement splints (MAS). These are inserted at night and are thought to act by displacing the position of the mandible and/or tongue with the aim of enlarging the pharyngeal airway or otherwise reducing its collapsibility. ${ }^{15}$

The majority of studies on the efficacy of mandibular advancement splints are case reports or series ${ }^{16}$ although recent publications report on prospective controlled trials investigating the differences between MAS and other treatment modalities. ${ }^{17-21}$

Snoring improves or is eliminated in the majority of patients treated with mandibular advancement splint therapy. ${ }^{16,22}$ Most studies rely on subjective reports by the patient or bed partner. Objective reduction in snoring by way of a decrease in the number of snores per hour, time spent snoring and mean sound level across the night has been demonstrated. ${ }^{23,24}$ The study by Johnston et $a .^{22}$ demonstrated the mandibular advancement splint as being significantly more effective than a placebo in reducing the frequency and loudness of snoring.

Objective data on the effect of mandibular advancement splints in obstructive sleep apnoea are determined by repeat sleep studies with the appliance in place. Findings vary according to the appliance used in the study and research methodology. The review of literature by Schmidt-Nowara et al. ${ }^{16}$ found a 50\% reduction in $70 \%$ of the 271 cases involved; however only 51\% reached a normal value (respiratory disturbance index $<10$ ); some did not improve or became worse; and 39\% with a baseline respiratory disturbance index of $>20$ remained above that level. Treatment success was related to the initial respiratory disturbance index in three studies reviewed and this has been reported by other authors, ${ }^{25,26}$ although this is not a consistent finding. Increase in respiratory disturbance index scores with mandibular advancement splint therapy has also been substantiated by more recent studies. ${ }^{26}$ Sleep and sleepiness have been assessed objectively by polysomnographic assessment. ${ }^{16}$ A reduction in sleep fragmentation, mid-sleep wake time and arousals has been demonstrated. Subjective improvement is an almost consistent finding ${ }^{25,27}$ and may be present in the absence of improvement in respiratory disturbance index. ${ }^{26}$

The present study is a retrospective report on the effects of mandibular advancement splints in two distinct subject groups, non-apnoeic snorers and subjects with obstructive sleep apnoea, using subjective and objective outcome measures.

\section{MATERIALS AND METHODS \\ Subjects}

The subjects for this retrospective study comprised adults referred from the Sleep Clinic to the Orthodontic Department of the Queen Victoria Hospital, East Grinstead, for construction of a mandibular advancement splint (MAS) to address their sleep-related breathing disorder. Each subject had undergone an overnight sleep study within the Sleep Studies Unit and completed an Epworth Sleepiness Scale ${ }^{10}$ (ESS), in order to establish the diagnosis and severity of their disorder. A diagnosis of obstructive sleep apnoea (OSA) was made following the American Academy of Sleep Medicine guidelines, ${ }^{5}$ that is if the Respiratory Disturbance Index (RDI) was greater 
than or equal to five. Patients with RDI less than five were diagnosed as non-apnoiec snorers.

Baseline data relating to demographic, anthropometric and severity of sleep-related breathing disorder are presented in Table 1. The subject's height and weight were measured in order to calculate the body mass index [BMI: weight $(\mathrm{kg}) /$ height $\left.\left(\mathrm{m}^{2}\right)\right]$. All the subjects underwent a full dental examination in order to determine the general dental condition and specifically the periodontal health. Patients were not judged suitable for mandibular advancement splint therapy if their dental status or periodontal health precluded them from wearing an appliance or if they were edentulous. All subjects were assessed and fitted with the appliance by one clinician (LW) between May 1997 and March 2000. Subjects were then invited to undergo a repeat sleep study with their mandibular advancement splint in situ and to complete a follow-up Epworth Sleepiness Scale and outcome questionnaire (Appendix 1).

On the basis of complete records being available, a total of 26 subjects (20 with obstructive sleep apnoea and six non-apnoeic snorers) who underwent treatment with a mandibular advancement splint, were retrospectively analysed.

\section{The mandibular advancement splint (MAS)}

Alginate impressions of the upper and lower dentition and an interocclusal wax registration, recorded in a position of maximum comfortable mandibular protrusion were obtained. A removable monobloc design was used (Fig. 1). This consisted of a one-piece hard acrylic splint incorporating full occlusal coverage and an opening anteriorly to allow for mouth breathing. Additional retention was obtained from Adam's cribs on the upper and lower first premolars and the upper first molars. The MAS design did not allow for any incremental mandibular advancement, and as such, it was not possible to adjust the degree of mandibular protrusion without remaking the appliance. Patients were instructed to wear the appliance at night-time only.

\section{Outcome measures and questionnaire design Sleep study}

Limited overnight sleep studies using the Edentrace monitoring system were performed within the Sleep Studies Unit at the Queen Victoria Hospital. Assessment was made of the following parameters: pulse oximetry, heart rate, nasal/oral airflow, chest impedance, posture and laryngeal noise. A typical output is seen in Figure 2, demonstrating the changes in these parameters at baseline and after MAS therapy. All subjects in the study underwent two overnight sleep studies, the first established a diagnosis of their sleep-related breathing disorder (baseline) and the second was designed to objectively assess the effect of their mandibular advancement splint. The latter study was undertaken after it had been ensured that the patient was comfortably wearing the splint every night and was minimally performed after one month of wear.

The respiratory disturbance index was calculated for each patient A baseline respiratory disturbance index (RDI) score of below five was used to distinguish non-apnoeic snorers from those with obstructive sleep apnoea. ${ }^{5}$ Success with mandibular advancement splint therapy, in subjects with OSA, was defined as a reduction in the RDI score to a value of ?5, as outlined by the American Academy of Sleep Medicine Task Force. ${ }^{5}$ Respiratory disturbance index scores after MAS therapy for the 26 patients allowed for analysis of treatment efficacy.

\section{Epworth Sleepiness Scale (ESS)}

All of the 26 subjects completed an Epworth Sleepiness Scale prior to fitting the appliance and at the time of the repeat sleep study.

\section{Outcome questionnaires}

A single operator (DA) attempted to contact all subjects by

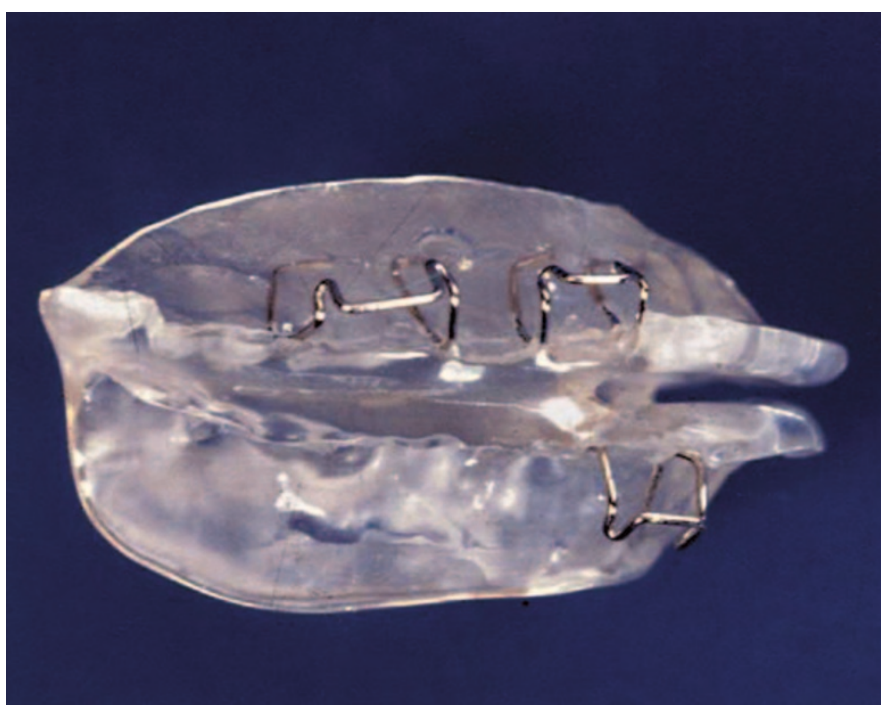

Fig. 1 The monobloc mandibular advancement splint

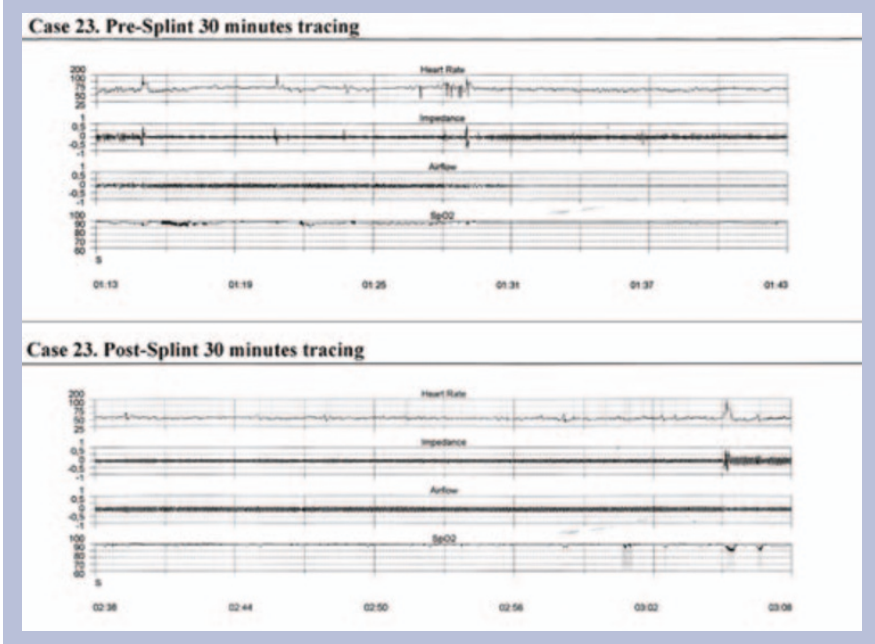

Fig. 2 Tracings from overnight sleep studies pre- and post-splint therapy

telephone to complete an outcome questionnaire following the repeat sleep study. The questionnaire (Appendix 1) was designed to assess snoring; sleepiness; complications of mandibular advancement splint therapy; the partner's experience and a comparison to nasal-CPAP, if relevant. Those subjects that were unavailable by telephone were sent the questionnaire by post.

\section{Statistical analysis}

Baseline respiratory disturbance index scores divided the sample into those with non-apnoeic snoring and those with obstructive sleep apnoea. Male/female frequency, median and range values for age and BMI, pre- and post-mandibular advancement splint therapy respiratory disturbance index and Epworth Sleepiness Scale scores were calculated for the obstructive sleep apnoea subjects and non-apnoeic snorers using SPSS (Statistical Package for Social Sciences, SPSS Inc., Chicago Ill, USA). Statistically significant differences in the RDI and ESS scores, before and after mandibular advancement splint therapy, were assessed using the Wilcoxon Signed Ranks test, at the 5\% level of significance.

\section{RESULTS}

\section{Subject demographics (Table 1)}

Twenty-six adult patients comprised the sample for this retrospective study, of which 20 (15 males; five females) were diagnosed with obstructive sleep apnoea (RDI ? 5) and six males with non-apnoeic snoring (RDI < 5). The median age of the OSA subjects was higher than that of the snorers (53.1 and 46.8 years 
respectively). Subjects in the non-apnoeic group were found to be slightly more overweight and demonstrated a higher level of daytime sleepiness (27 and 12.5, respectively) compared to the obstructive sleep apnoea subjects ( 28 and 10, respectively).

\section{Changes in severity (Table 2)}

Table 2 reports the changes in the subject's severity of sleeprelated breathing disorder following mandibular advancement splint therapy. All categories of obstructive sleep apnoea (mild, moderate, severe) improved as a result of treatment, with an overall reduction in the numbers of subjects with OSA, from 20 to 13. Thus, the numbers of subjects with non-apnoeic snoring increased from six to 13 as a result of intervention.

\section{Effects on outcome measures (Table 3 )}

No statistically significant change was observed in the Epworth Sleepiness Scale scores in either subject group. However, subjects with obstructive sleep apnoea demonstrated a reduction in median Respiratory Disturbance Index scores from 18 to $7(\mathrm{P}<0.05)$ following mandibular advancement splint therapy.

Twenty-one of the 26 subjects completed the outcome questionnaire thus obtaining a compliance rate of 81 per cent. Seventeen of these reported an improvement in snoring and sleep quality. The remaining four reported no difference. Side-effects were generally transient and included jaw ache on awakening, dry throat and ulceration. Eighty-six per cent of the subjects' partners reported better quality of sleep as a result of treatment.

\section{DISCUSSION}

Two of the most commonly used measures in assessing treatment outcomes in sleep-related breathing disorders are the Epworth sleepiness scale (ESS) and the respiratory disturbance index (RDI). Despite their subjective nature, questionnaire outcome measures are also widely used. ${ }^{16,22}$ Polysomnography is regarded as the 'gold standard' for diagnosing obstructive sleep apnoea (OSA) and is recommended for the evaluation of treatment efficacy in moderate to severe OSA subjects treated with mandibular advancement splints. ${ }^{13}$ However, the technique is expensive and not readily available, requiring a special sleep laboratory and trained technical support. The sleep study used in this report measured oxygen saturation, heart rate, airflow, chest movement and laryngeal noise. Stradling and Crosby ${ }^{28}$ found a close correlation of $4 \%$ oxygen dips with the apnoea index. Douglas et al. ${ }^{29}$ found oximetry, time in bed, breathing pattern and thoraco-abdominal and leg movement to be of value in diagnosing OSA. One of the limitations of this assessment within the sleep laboratory is the lack of familiarity of the surroundings and presence of the multitude of probes which can affect sleep. This may mean the resultant respiratory disturbance index is not representative of the subject's condition. Home monitoring pre- and post-splint may have been an alternative method of assessing mandibular advancement splint efficacy although this potentially could have introduced problems with subject compliance and operating the equipment.

There is currently no universally agreed definition of treatment success for obstructive sleep apnoea (OSA). Authors have reported a $50 \%$ reduction in respiratory disturbance index score or alternatively, a reduction in the absolute respiratory disturbance index value to less than or equal to $10 .{ }^{16}$ Treatment success in this study was defined as a reduction in respiratory disturbance index to below five, in order to meet the definitions outlined by the American Academy of Sleep Medicine Task Force. ${ }^{5}$ Forty per cent of the OSA subjects achieved this. This compares very favourably with the findings of Johnston et al. ${ }^{30}$ in which a success rate of 33\% was reported when using a similar design of mandibular advancement splint. However, these authors defined success as a reduction of the RDI score to 10 or below and should therefore have reported slightly better success rates. The authors partly attributed the low success rate to the inclusion of severe obstructive sleep apnoea subjects (RDI greater than 50) in their sample. Several studies have found an association between baseline respiratory disturbance index and treatment response with a low initial respiratory disturbance index being a good predictor of treatment success. ${ }^{21,25,26}$ This was not consistent in this study; two severe obstructive sleep apnoea subjects were considered treatment successes and two mild obstructive sleep apnoea subjects became severe obstructive sleep apnoea subjects. Of the eight successes however, five were mild OSA subjects prior to treatment. Thus, the effects of mandibular advancement splint therapy on sleep-related breathing disorders appear to be more complicated than simply assigning success directly to the initial respiratory disturbance index score.

All subjects completed a validated questionnaire designed to assess daytime sleepiness. ${ }^{10}$ The relatively low median Epworth Sleepiness Scale (ESS) scores in the obstructive sleep apnoea group (10, range 1 to 18 ) is in contrast to other studies using the ESS, where OSA patients have mean values of $16 .{ }^{31}$ This suggests that in the current study, the OSA subjects were not regarded as showing excessive baseline daytime sleepiness. ${ }^{10}$ This may explain the nonsignificant improvement in ESS scores in the face of the significant reduction in the respiratory disturbance index in this group. This was also observed by Johnston et al. ${ }^{30}$

The non-apnoeic snoring group $(\mathrm{n}=6)$ were by definition, subjects with an initial respiratory disturbance index score of less than five episodes of abnormal breathing per hour of sleep. ${ }^{5}$ Thus, repeat RDI scores could not measure success directly and were of specific value in determining if the splint worsened their sleeprelated breathing disorder. This was the case in one subject in the present study who became categorised as a mild obstructive sleep apnoea subject. The risk of exacerbating the sleep-related breathing disorder by mandibular advancement splint therapy has been previously reported and indeed is the reasoning behind undertaking a follow-up objective assessment. . $^{23,32,33}$

Mandibular advancement splints are thought to act by increasing the size and/or reducing the collapsibility of the pharyngeal airway. ${ }^{15}$ Cephalometric predictors have been suggested where a favourable response to MAS therapy can be expected ${ }^{15}$ but the technique at best only provides a two-dimensional image. Correlation with the cephalometric data was not carried out in this study but may have shown contraindications in the subjects that did not respond favourably. Studies of the effects of mandibular protrusion on the airway have also shed some light on the variability of response to MAS therapy. ${ }^{32}$ Mandibular opening has been shown to occlude the oropharyngeal airway in subjects with increased vertical facial proportions and retrognathic mandible. ${ }^{32}$ Ryan et al. ${ }^{35}$ found the lateral diameter of the velopharynx was the airway site that changed the most with mandibular advancement splints. It may be that the nonresponders in this study had obstruction, either high in the nasopharynx or very low in the pharynx beyond the therapeutic range of the splint. Johal and Battagel ${ }^{15}$ suggested mandibular advancement splint therapy is of little use in those OSA subjects who exhibit primarily palatal level obstruction based on the findings of Pringle and Croft. ${ }^{36}$ Rodenstein et al. ${ }^{37}$ found the pharynx to be circular or elliptical with the long axis oriented in the sagittal plane in OSA subjects as opposed to the coronal plane in normal subjects. With mandibular protrusion it is possible that the walls of the pharynx are pulled closer together in some OSA subjects thus adding to the obstruction and negating the effects of the appliance. This may explain the observed increase in the RDI scores at follow-up in the current study. Furthermore, it has been demonstrated that the shape of the pharyngeal lumen is more dependent on the body mass index (BMI) than on the presence of OSA. ${ }^{38}$ All subjects in the current study demonstrated higher than 'normal' values of BMI and this may have influenced the effect of mandibular advancement splint therapy. 
The amount of protrusion necessary for an adequate response has been reported as 75 per cent of the maximum protrusion..$^{25,34}$ Lowe et al. ${ }^{26}$ reported increased treatment success with a titratable appliance that was adjusted according to the subject's symptoms to a maximum of $85 \%$ of maximum protrusion. A balance needs to be met such that the protrusion required to improve the airway does not result in mandibular opening of sufficient magnitude to negate this effect. In this study, the monobloc appliance was made such that the patient was at maximum comfortable protrusion although this may have resulted in mandibular opening. The appliance design did suffer the disadvantages of not permitting progressive mandibular advancement and, as such, may not have achieved its optimal therapeutic benefit. Furthermore, there is increased vertical opening associated with this form of splint design. ${ }^{30}$

The group of non-apnoeic snorers had a median baseline Epworth sleepiness scale score of 12.5 indicating that these subjects had increased levels of daytime sleepiness despite, by definition, not suffering from repeated desaturation and resultant arousals. This score reduced to 8.5 following mandibular advancement splint therapy and is consistent with the findings of Johnston et al. ${ }^{22}$ This supports the idea of a continuum of levels of sleep apnoea rather than definitive diagnoses based solely on the polysomnographic findings.

Eighty-six per cent of the subjects' partners reported better sleep quality as a result of mandibular splint therapy. This outcome measure has been used by several authors ${ }^{16,22}$ with similarly encouraging results.

\section{Limitations of the study}

The limitations of this retrospective study were that only subjects with complete records were evaluated for treatment effect and as such, bias cannot be excluded. The sleep study results, both at baseline and following splint therapy, may not have been representative of a subject's regular night's sleep. However, no single instrument exists, to date, that overcomes this limitation.

The use of a post-treatment evaluation questionnaire relied on the subjects being contactable by post and/or telephone. Better evaluation of the subjective assessment may have been possible by inviting the subjects to attend for an interview. However, an acceptable compliance rate of $81 \%$ was achieved.

The mandibular advancement splint used in this study did not permit further advancement, without the remaking of the splint, thus it was not possible to titrate the necessary advancement with the subject's response and tolerance.

\section{CONCLUSIONS}

The monobloc appliance significantly improved the respiratory disturbance index in the obstructive sleep apnoea subjects.

Eighty-six per cent of the subject's partners reported better quality of sleep following mandibular advancement splint therapy.

Some subjects had increased respiratory disturbance index scores following splint therapy. This highlights the need for an objective assessment in the follow-up of patients treated with mandibular advancement splints.

1. Lavie P. Nothing new under the moon: historical accounts of sleep apnea syndrome. Arch Intern Med 1984; 144: 2025-2028.

2. Lévy P, Pepin J-L, Mayer P, Wuyam B, Veale D. Management of simple snoring, upper airway resistance syndrome, and moderate sleep apnea syndrome. Sleep 1996; 19:S101-110.

3. Brown L K. Sleep apnea syndromes: overview and diagnostic approach. Mt Sinai Medicine 1994; 61: 99-112.

4. Stradling J R. Obstructive sleep apnoea: definitions, epidemiology, and natural history. Thorax 1995; 50: 683-689.

5. American Academy of Sleep Medicine Task Force. Sleep-related breathing disorders in adults: recommendations for syndrome definition and measurement techniques in clinical research. Sleep 1999; 18: 677-689.

6. Ohayon M M, Guilleminault $C$, Priest R G, Caulet M. Snoring and breathing pauses during sleep: telephone interview survey of a United Kingdom population sample. BMJ 1997; 314: 860-863.
7. Gibson G J, Douglas N J, Stradling J R, London D R, Semple S J. Sleep apnoea: Clinical importance and facilities for investigation and treatment in the UK. Addendum to the 1993 Royal College of Physicians of London Sleep Apnoea report. J R Coll Phys Lond 1998; 32: 540-544.

8. Wright J, Johns $\mathrm{R}$, Watt I, Melville A, Sheldon T. Health effects of obstructive sleep apnoea and the effectiveness of continuous positive airways pressure: a systematic review of the research evidence. BrMed J 1997; 314: 851-860.

9. Emsellem H A, Corson W A, Rappaport B A, Hackett S, Smith L G, Mausfeld J N. Verification of sleep apnoea using a portable sleep apnoea screening device. Southern Medical Journal 1990: 83: 748-752.

10. Johns M W. Daytime sleepiness, snoring, and obstructive sleep apnea The Epworth sleepiness scale. Chest 1993; 103: 30-36

11. Johns M W. A new method for measuring daytime sleepiness: the Epworth Sleepiness Scale. Sleep 1991; 14: 540-545

12. Osman E Z, Osborne J, Hill P D, Lee B W. The Epworth Sleepiness Scale: can it be used for sleep apnoea screening among snorers? Clin Otolaryngo/ 1999; 24: 239-241.

13. American Sleep Disorders Association Report. Practice parameters for the treatment of snoring and obstructive sleep apnea with oral appliances. Sleep 1995; 18: 511-513.

14. Johal A. The relationship between obstructive sleep apnoea and dentistry: 2 . Management. Dent Update 1998; 25: 474-477.

15. Johal A, Battagel J M. Current principles in the management of obstructive sleep apnoea with mandibular advancement appliances. Br DentJ 2001; 190: 532-536.

16. Schmidt-Nowara W W, Lowe A, Wiegand L, Cartwright R, Perez-Guerra F, Menn S. Oral appliances for the treatment of snoring and obstructive sleep apnea: a review. Sleep 1995; 186: 501-510.

17. Clark G T, Blumenfeld I, Yoffe N, Peled E, Lavie P. A crossover study comparing the efficacy of continuous positive airway pressure with anterior mandibular positioning devices on patients with obstructive sleep apnea. Chest 1996; 109: 1477-1483.

18. Ferguson $K$ A, Ono $T$, Lowe A A, al-Majed $S$, Love $L$ L, Fleetham J A. A short-term controlled trial of an adjustable oral appliance for the treatment of mild to moderate obstructive sleep apnoea. Thorax 1997; 52:362-368.

19. Lamont J, Baldwin D R, Hay K D, Veale A G. Effect of two types of mandibular advancement splints on snoring and obstructive sleep apnoea. Eur J Orthod 1998; 20: 293-297

20. Walker-Engström M L, Wilhelmsson B, Tegelberg A, Dimenas E, Ringqvist I. Quality of life assessment of treatment with dental appliance or UPPP in patients with mild to moderate obstructive sleep apnoea. A prospective randomized 1-year follow-up study. $J$ Sleep Res 2000; 9: 303-308.

21. Tan Y K, L'Estrange P R, Luo Y M et al. Mandibular advancement splints and continuous positive airway pressure in patients with obstructive sleep apnoea: a randomized crossover trial. Eur J Orthod 2002; 24: 239-249.

22. Johnston C D, Gleadhill I C, Cinnamond M J, Peden W M. Oral appliances for the management of severe snoring: a randomized controlled trial. Eur J Orthod 2001; 23: 127-133.

23. O'Sullivan R A, Hillman D R, Mateljan R, Pantin C, Finucane K E. Mandibular advancement splint: an approach to treat snoring and obstructive sleep apnea. Am J Respir Crit Care Med 1995; 151: 194-198.

24. Smith A-M. The effectiveness of mandibular advancement devices in subjects who snore MSc, University of London, 1999.

25. Liu Y, Zeng X, Fu M, Huang X, Lowe A A. 2000 Effects of a mandibular repositioner on obstructive sleep apnea. Am J Orthod Dentofacial Orthop 118: 248-256.

26. Lowe A A, Sjoholm T T, Ryan C F, Fleetham J A, Ferguson K A, Remmers J E. Treatment, airway and compliance effects of a titratable oral appliance. Sleep 2000; 23: $\mathrm{S} 172-178$

27. Bernhold $M$, Bondemark L. A magnetic appliance for treatment of snoring patients with and without obstructive sleep apnea. Am J Orthod Dentofac Orthop 1998; 113: 144-155.

28. Stradling $J \mathrm{R}, \mathrm{Crosby} J \mathrm{H}$. Predictors and prevalence of obstructive sleep apnoea and snoring in 1001 middle aged men. Thorax 1991; 46: 85-90.

29. Douglas N J, Thomas S, Jan M A. Clinical value of polysomnography. Lancet 1992; 339 : 347-350.

30. Johnston C D, Gleadhill I C, Cinnamond M J, Gabbey J, Burden D J. Mandibular advancement appliances and obstructive sleep apnoea: a randomized clinical trial. Eur J Orthod 2002: 24: 251-262.

31. Bennett LS, Davies R J O, Stradling J R. Oral appliances for the management of snoring and obstructive sleep apnoea. Thorax 1998; 52: 362-368.

32. L'Estrange P R, Battagel J M, Harkness B, Spratley M H, Nolan P J, Jorgensen G I. A method of studying adaptive changes of the oropharynx to variation in mandibular position in patients with obstructive sleep apnoea. J Oral Rehabil 1996b; 23: 699-711.

33. Gale D J, Sawyer R H, Woodcock A, Stone P, Thompson R, O'Brien K. Do oral appliances enlarge the airway in patients with obstructive sleep apnoea? A prospective computerized tomographic study. Eur J Orthod 2000; 22: 159-168.

34. Battagel J M, Johal A, Kotecha B. A cephalometric comparison of subjects with snoring and obstructive sleep apnoea. Eur J Orthod 2000; 22: 353-365.

35. Ryan C F, Love L L, Peat D, Fleetham J A, Lowe A A. Mandibular advancement ora appliance therapy for obstructive sleep apnoea: effect on awake calibre of the velopharynx. Thorax 1999; 54: 972-977.

36. Pringle $M B$, Croft $C B$. A grading system for patients with obstructive sleep apnoea based on sleep nasendoscopy. Clin Otolaryngol 1993; 18: 480-484.

37. Rodenstein D O, Dooms G, Thomas Y, Liistro G, Stanescu D C, Culee C, Aubert-Tulkens G. Pharyngeal shape and dimensions in healthy subjects, snorers, and patients with obstructive sleep apnoea. Thorax 1990; 45: 722-727.

38. Mayer P, Pepin J L, Bettega G, Veale D, Ferretti G, Deschaux C, Levy P. Relationship between body-mass index, age and upper airway measurements in snorers and sleep-apnoea patients. Eur Respir J 1996; 9: 1801-1809. 


\section{Appendix 1 THE QUEEN VICTORIA HOSPITAL - Questionnaire of Intraoral Mandibular Advancement Splint}

Name:

DOB:

1. Are you still using your splint:

2. If not using the splint, when did you stop and why?

3. How many nights per week do you use the splint?

4. Since treatment are you snoring?
Home Phone No:

Work Phone No:

Yes $\square$

No $\square$

$\square$ more than before
$\square \quad$ as much as before
$\square \quad$ less than before
$\square$ not at all
$\square$ don't know

5. Since using the splint do you have any of the following problems: aching teeth

\begin{tabular}{|c|c|c|}
\hline Yes $\square$ & No $\square$ & Improved $\square$ \\
\hline Yes $\square$ & No $\square$ & Improved $\square$ \\
\hline Yes $\square$ & No $\square$ & Improved $\square$ \\
\hline Yes $\square$ & No $\square$ & \\
\hline Yes $\square$ & No $\square$ & \\
\hline Yes $\square$ & No $\square$ & Don't know $\square$ \\
\hline$\square$ using the splint? & $\square$ not using the splint? & \\
\hline \multicolumn{3}{|c|}{$\square$ better than it was before treatment. } \\
\hline \multicolumn{3}{|c|}{$\square$ the same as it was before treatment. } \\
\hline \multicolumn{3}{|c|}{$\square$ worse than it was before treatment. } \\
\hline Yes $\square$ & No $\square$ & \\
\hline Yes $\square$ & No $\square$ & \\
\hline \multicolumn{3}{|c|}{$\square$ better than it was before treatment. } \\
\hline \multicolumn{3}{|c|}{$\square$ the same as it was before treatment. } \\
\hline \multicolumn{3}{|l|}{ than it wa } \\
\hline
\end{tabular}

12. When you wake in the morning do you suffer from:

headache?

dry throat?

sore throat?

nasal congestion?

being refreshed?

Yes $\square$
Yes $\square$
Yes $\square$
Yes $\square$
Yes $\square$
$\square \quad$ better than before?
$\square \quad$ worse than before?
$\square \quad$ same as before?

14. Was the sleep study report sent to you helpful?

Yes $\square$

No $\square$

No $\square$

No $\square$

No $\square$

No $\square$

13. Is your general feeling of well being using the splint:
$\square$ worse than before?
same as before?

15. If you have used CPAP, in comparison, would you say the splint is: more effective more convenient

better tolerated

more acceptable

to your partner

e

Any other comments? 